\title{
Los diccionarios inversos de la lengua española: Descripción del repertorio bibliográfico
}

\author{
Susana Guerrero Salazar \\ Universidad de Málaga
}

\section{INTRODUCCIÓN}

Hace ya unos años, Gabriel María Verd (1993) publicó un artículo en el que por primera vez se llevaba a cabo una investigación lexicográfica seria sobre los diccionarios inversos españoles que habían visto la luz hasta el año 1991, indicando sus aciertos y errores. En primer lugar, el autor llamaba la atención sobre la utilidad de este producto lexicográfico, que en otros idiomas se conoce desde antiguo y se ha utilizado para el conocimiento de la lengua en distintas etapas y en diversos campos. En segundo lugar, dejaba claro su origen, pues, aunque durante mucho tiempo el nacimiento de los diccionarios inversos se ha situado a finales del XIX, existen desde el siglo XVI' ${ }^{1}$. En tercer lugar, presentaba la descripción de los diccionarios y, por último, manifestaba la necesidad de confeccionar diccionarios onomásticos inversos.

El propósito de este trabajo es completar el iniciado por Verd, sobre todo en dos aspectos: por un lado, fijaré las características de este tipo de diccionario, que, como veremos, unas veces se confunde con otros

1 Según Verd (1993) el primer diccionario inverso es el bilingüe alemán-latín de Erasmus Alberus, Novum Dictionarii Genus, in quo ultimis seu terminalibus Germanicarum vocum syllabis observatis Latina vocabula, cum suis quaeque synonymis [...] protinus sese offerunt, Frackfurt Chr. Egenolphus (1540, reimpreso en 1565 y 1975); en 1880 se publica el de Schäfer, también del alemán y el de H. Hoogeveen, del griego (reeditado en 1810); en $1836 \mathrm{~W}$. Pape publica otro del griego; a finales del XIX (1873-1875) sale a la luz el que casi todo el mundo cita como el primer diccionario inverso, el védico de H. Grapmann, Wörterbuch zur Rig-véda, Leipzig, 1973. 
repertorios lexicográficos (como son los diccionarios de la rima) y otras se utiliza su nombre para designar obras que en absoluto tienen nada que ver con un diccionario inverso. Por otro lado, completaré la descripción de los diccionarios inversos publicados en español hasta el momento.

\section{DESCONOCIMIENTO Y CONFUSIÓN CON RESPECTO AL DICCIONARIO INVERSO}

El diccionario inverso, llamado también de terminaciones o con el anglicismo innecesario reverso, es todavía un gran desconocido. Quizás a ello se debe el que se utilice erróneamente el término inverso para designar productos lexicográficos muy dispares. Tal es lo que ocurre, por ejemplo, con el Diccionario inverso ilustrado. De la idea aproximada a la palabra precisa (1997), que mantiene el orden alfabético usual $^{2}$. Otro caso es el apéndice denominado Diccionario inverso que se incluye en El diccionario de eufemismos de Lechado García (2000), que consiste en definir el término o expresión más directo por medio de sus equivalencias eufemísticas.

Además de la confusión terminológica, existe -con frecuencia incluso entre los especialistas- la tendencia a creer que el diccionario de la rima y el inverso son una misma cosa, debido, en gran parte, al desconocimiento. Esta confusión se puede explicar porque son diccionarios específicos, que han tenido una escasa distribución y se hallan muy dispersos $^{3}$. Sin embargo, estos dos productos lexicográficos nacen en mo-

2 Se trata de una especie de diccionario ideológico en cuanto que tiene como finalidad localizar el término preciso (palabra-objetivo) a partir de una idea (palabra-clave), mediante las definiciones, las referencias a otros términos relacionados (sinónimos, palabras afines y antónimos) y las ilustraciones.

3 Curiosamente, en la definición que se aporta en el prólogo del diccionario reverso de David Mighetto y Per Rosengren (1985) se mezclan los dos tipos: "Los diccionarios reversos -también llamados diccionarios inversos o de la rima- se alfabetizan de derecha a izquierda comenzando por el final de la palabra". Esta definición es válida para los diccionarios inversos pero no para los de la rima. En el prólogo del diccionario inverso de Enrique Fontanillo y $\mathrm{M}^{\mathrm{a}}$ Isabel Riesco (1992), no sólo se confunden am- 
mentos históricos diferentes; hacen de instrumentos auxiliares en materias muy distintas; cada uno posee un funcionamiento peculiar, y sus finalidades difieren en gran parte. Veamos brevemente las diferencias:

En primer lugar, el nacimiento del diccionario de la rima es bastante anterior al del inverso, ya que el primero surge ligado a las preceptivas retóricas en el siglo XIV, mientras que el diccionario inverso nace en el siglo XVI, cobra auge en el XIX -ligado, sobre todo, al estudio de las lenguas muertas ${ }^{4}$ - y es ya entrado este siglo cuando conoce la aplicación a las lenguas modernas 5 . En lengua española, la distancia entre los dos tipos de diccionarios es de cinco siglos: el primer diccionario de la rima data del XV, La Gaya Ciencia de Pedro Guillén de Segovia (1475) y el primer diccionario inverso del XX, A Reverse Dictionary of the Spanish Language de Fred A. Stahl y Gary E. A. Scavnicky (1973).

En segundo lugar, aunque tanto los diccionarios de la rima como los

bos tipos de diccionarios, sino que se considera a los diccionarios de la rima los predecesores de los inversos y se fecha el nacimiento de estos en el siglo pasado, cuando existen desde el XVI: "Los diccionarios inversos, es decir, aquellos que, como este, nos dan las palabras ordenadas alfabéticamente de final a principio, existen desde el siglo XIX, y su primera aplicación fue la de ayudar a los versificadores a encontrar términos que resolvieran sus problemas de rima. No se llamaban inversos ni eran exactamente lo que ahora entendemos como tales, pero pueden, perfectamente, considerarse sus predecesores". Sobre los afinidades y diferencias entre los diccionarios inversos y los de la rima, véase Guerrero Salazar 2001a; sobre los diccionarios de la rima del español, véase Guerrero Salazar 2001b y 2002.

4 Los diccionarios inversos se usaron como instrumento auxiliar para los investigadores, arqueólogos, filólogos, etc., interesados por los documentos de la antigüedad, para lo que resulta muy interesante tabular el vocabulario de las lenguas clásicas, ordenándolo alfabética y estrictamente por los finales de cada palabra. De hecho, los diccionarios inversos facilitan la lectura y reconstrucción de textos manuscritos antiguos, inscripciones, etc., que poseen gran cantidad de palabras truncadas. Por esta razón, los primeros diccionarios inversos están relacionados con las lenguas sánscritas, latina, paleoiránica, griega y paleoeslávica eclesiástica.

5 El primer diccionario inverso en lengua moderna fue publicado por la Academia $\mathrm{Ru}$ mana en Bucarest y apareció en 1957. A partir de éste se fueron publicando otros en todas las lenguas modernas importantes, algunos especializados en etapas concretas, otros trabajan con corpus especiales, según finalidades científicas o didácticas, etc. Véase el esbozo histórico al respecto de Jitka Stindlova (1960). 
diccionarios inversos presentan unos listados de palabras distribuidas en columnas y agrupadas por terminaciones ${ }^{6}$, las diferencias entre estos dos tipos de diccionarios son evidentes:

a) Los diccionarios inversos se alfabetizan de derecha a izquierda, comenzando por el final de la palabra y no por la vocal acentuada, como los de la rima.

b) En los diccionarios inversos las palabras se agrupan según tengan igual terminación, prescindiendo del acento, ya que lo que importa es la forma gráfica y no la pronunciación de las unidades léxicas, por ello, no hay separación entre agudas, graves y esdrújulas, como suele ocurrir en los de la rima.

c) Los diccionarios inversos se limitan a ordenar los términos según sus terminaciones, por ello no suelen agrupar las palabras por el número de sílabas, lo que resulta bastante útil en los diccionarios de la rima (aunque no todos lo hagan) ${ }^{7}$.

d) Dado que el diccionario inverso es un producto lexicográfico más moderno que el diccionario de la rima, los diccionarios inversos del español se han beneficiado de los procedimientos informáticos y de los avances de la lexicografía; por ello, aparecen estrechamente vinculados a los índices de frecuencia, las concordancias léxicas, etc.

e) Los diccionarios inversos han absorbido, en cierto modo, la aplicación de los diccionarios de la rima, ya que, además de servir de instrumento para poetas y versificadores, también aportan una utilidad lúdica, publicitaria, ortográfica, etimológica, semántica e investigadora (morfología derivativa, fonología, lingüística computacional, estudios comparativos, etc.).

6 Otra afinidad entre diccionarios inversos y de la rima es que en ninguno de ellos la información gramático lexical es importante, aunque puede aparecer.

7 El poeta con frecuencia busca no sólo una palabra con una terminación concreta, sino también con un número de sílabas determinado. 


\section{LOS DICCIONARIOS INVERSOS EN LENGUA ESPAÑOLA}

En cuanto a los diccionarios inversos en lengua española, los primeros tuvieron escasa difusión dado que se publicaron en el extranjero, como el primero, de Stahl y Scavnicky (Estados Unidos, 1973), el de Mighetto y Rosengren (Suecia, 1985), el de S. Faitelson-Weiser (Quebec, 1987); otros fueron fruto de ediciones privadas, como el de Martínez y Martínez de Carnero (1978). Sin embargo, a partir de 1987, la publicación de este tipo de diccionarios aumenta considerablemente. Sólo en ese año se publicaron tres: el Diccionario Inverso del español. Su uso en el aula, de Hermenegildo de la Campa, el Dictionnaire inverse et analyse statistique de la langue espagnole, de Silvia FaitelsonWeiser y el Diccionario inverso de la lengua española, de Ignacio Bosque y Manuel Pérez Fernández. Desde 1987 a 1996, al menos se han publicado seis diccionarios más. No todos toman como base las mismas fuentes, ni persiguen las mismas finalidades.

A continuación, haré un breve recorrido a través de los diccionarios inversos en lengua española, respetando el orden cronológico de publicación, completando así el repertorio bibliográfico iniciado por Verd (1993).

\subsection{A ReVERSE Dictionary of the SPANish LANGUAGE DE F. A. STAHL Y G. E. A. SCAVNICKY (1973)}

Curiosamente, el primer diccionario inverso de la lengua española se publicó en la Universidad de Illinois y tanto el prólogo (foreword) como el prefacio (preface) están escritos en lengua inglesa. El diccionario consta de 181 páginas y contiene alrededor de 86.081 voces extraídas de la decimoctava edición del diccionario académico de 1956. Está precedido de un prólogo en el que J. H. D. Allen explica las dos razones principales por la que estos diccionarios no han sido lo suficientemente reconocidos:

The first of these is the ill-founded suspicion, rather widespread even among humanists, that the term refers to a dictionary constructed by placing at the beginning all words with initial Z-, and at the end all words with initial A-, with the remainder appropriately orde- 
red between. A glance at Stahl and Scavnicky's text will quickly reveal that this is not the case; yet not probably the obvious lack of utility in such a procedure causes much of the skepticism with which mention of these works is often greeted among humanists.

The second reason for the neglect of these dictionaries is of a different kind. The labor of producing one, if it is done in the conventional way with pencil and paper, is so enormous that even the most intrepid scholars may easily be dissuaded from undertaking it. As a consequence, until quite recently very few such dictionaries have been compiled and published, and there must be numerous serious students of language who have not yet seen one.

A continuación, el prologuista nos advierte de las ventajas de los grandes ordenadores para los estudios lingüísticos y, particularmente, lexicográficos. Reconoce, además, que, gracias a la informática, la publicación de estos trabajos se ha visto sustancialmente enriquecida y aprovechada para el estudio de aspectos que superan los objetos iniciales, pues, en principio, este tipo de diccionarios se relacionaba tan solo con las lenguas clásicas y la reconstrucción de textos truncados. Ahora, sin embargo, se puede estudiar, por ejemplo, la productividad de determinados sufijos:

The process of reverse ordering obviously brings together (in many languages) classes of words such as verbs of the same conjugation and words formed with the same suffix, e.g., in Spanish verbs in -izar, adverbs in -mente, adjetives in -oso, -doso, -dadoso, and abstract nouns in - $d a d$. Thus a great deal of the useless labor is removed from the study of the use of suffixes to make new formations, the growth or diminution of the vitality of particular suffixes, preferences for one of two or more similar suffixes (e.g., the Spanish diminutives -ito and -ico) and other investigations of the final parts of words. The usefulness of reverse ordering subsumes, but is not limited to, most of the uses of the more familiar rhyming dictionaries.

El prólogo termina elogiando la labor de Stahl y Scavnicky, cuyo diccionario viene a llenar una carencia en la lengua española, y, en este sentido, sigue el ejemplo de otras lenguas romances que ya en este momento poseían diccionario inverso (rumana, 1957; italiana, 1962 y francesa, 1963). 
Seguidamente, en el breve prefacio, los autores explican el procedimiento informático seguido y cómo han solucionado el problema de la $\tilde{n}$, los acentos y la ordenación de la $c h$ y de la $l l$; esta última cuestión no ha sido mantenida en otros diccionarios inversos (que han resuelto el problema considerando los dígrafos como dos letras independientes).

En cuanto al diccionario propiamente dicho, consiste en un listado de palabras escritas en mayúsculas, ordenadas alfabéticamente a partir de los finales, las cuales se distribuyen en cinco columnas. No hay indicaciones gramaticales, ni apéndices, ni estadísticas. Tan sólo se indica la letra final, en torno a la cual se agrupan los términos. Veamos como ejemplo las primeras palabras de la letra $\mathrm{N}$ :

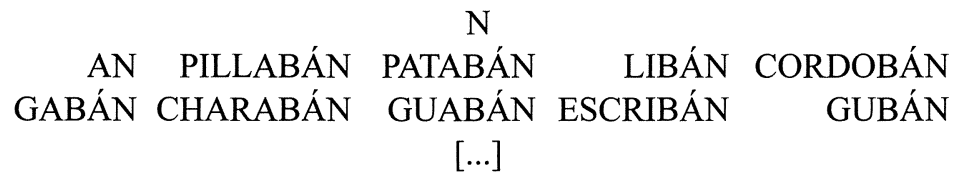

3.2. DiCCIONARIO DE TERMINACIONES DE J. J. MARTíNEZ Y MARTíNEZ DE CARNERo (1978)

Este diccionario de terminaciones es el más pequeño en cuanto al tamaño; viene a ser como un libro de bolsillo. Basado en la decimonovena edición del DRAE (1970), presenta 88.960 voces distribuidas en 371 páginas, en cuatro columnas y ordenadas alfabéticamente al revés.

No hay separación ninguna, el listado de palabras sigue el orden alfabético exhaustivo sin ninguna otra distinción, ni acentual, ni silábica ni gramatical:

$\begin{array}{rrrr}\text { MULO } & \text { EMULO } & \text { POMULO } & \text { ANULO } \\ \text { PICAMULO } & \text { TREMULO } & \text { CUMULO } & {[\ldots . .} \\ \text { FAMULO } & \text { DISIMULO } & \text { TUMULO } & \\ \text { CALPAMULO } & \text { ESTIMULO } & \text { NULO } & \end{array}$

En la breve introducción el autor menciona las utilidades que este tipo de diccionarios puede prestar a la semántica, a la filología, a la poesía, a la enseñanza del español y a los aficionados a crucigramas y juegos similares. 


\subsection{DicCionario ReVERSo de D. Mighetto y P. Rosengren (1985)}

Este diccionario publicado en Suecia fue el primero en manejar un corpus fuera del DRAE; de hecho ofrece un inventario de 52.237 unidades que provienen íntegramente de textos españoles extraídos de la prensa $^{8}$. Nos llama la atención el anglicismo reverso para nombrarlo, cuando hubiera resultado más apropiado inverso o de terminaciones.

El listado está dispuesto en tres columnas siguiendo el orden alfabético al revés. Que el corpus provenga de la prensa justifica que el léxico, como comprobamos en el ejemplo, posea una gran variedad:

$\begin{array}{rrr}a b & \text { club } & \text { coñac } \\ \text { scab } & \text { cine-club } & \text { crac } \\ \text { lib } & \text { pub } & \text { frac } \\ \text { snob } & \text { sub } & a b c \\ \text { bistec } & \text { picnic } & \text { cinc } \\ \text { hic } & \text { tonic } & \text { zinc } \\ \text { ethic } & \text { scalextric } & \text { nunc } \\ \text { public } & \text { sir } & \text { hoc } \\ \text { cómic } & \text { music } & \text { roc } \\ \text { comic } & \text { tinc } & \end{array}$

$\mathrm{Al}$ dar cuenta de la lengua viva y actual, el diccionario omite palabras anticuadas y muertas que se encuentran en otros diccionarios inversos, pero incluye préstamos, sobre todo anglicismos, que dan cuentan de la invasión de éstos en los medios de comunicación actuales. Este diccionario es, por tanto, muy interesante para un estudio sincrónico de la lengua.

Dado que los diccionarios inversos atienden a la forma gráfica y no a la pronunciación, no se tiene en cuenta la posición del acento para ordenar las palabras, como podemos apreciar en el siguiente ejemplo:

8 El Diccionario reverso $(D R)$ se compone de 206 páginas. Las 52.237 palabras castellanas y palabras-cita están extraídas de Palabras Gráficas Españolas: Lista y frecuencia en Prensa Española, 1977 (Mighetto y Rosengren 1983) las cuales, a su vez, han sido obtenidas del Banco de datos de Prensa Española: Concordancia lingüística y texto fuente (Mighetto y Rosengren 1982). 
compañeras

peras

paperas

óperas toperas

ásperas

esperas

vísperas prósperas

azucareras

camareras

olivareras

El diccionario constituye un inventario de lectura $a$ tergo que agrupa términos con finales iguales. Tan sólo se introducen las formas flexivas de nombres y verbos.

En el prólogo se explica que el diccionario está dirigido a los interesados en la rima, en los juegos de lenguaje, en la investigación y en la enseñanza de la lengua, y que también puede ser una referencia y herramienta de trabajo para los hispanistas que se dedican a describir matizadamente estructuras morfonológicas en uso dentro del castellano escrito.

Las estadísticas que incluye este diccionario difieren de las de los diccionarios de Hermenegildo de la Campa y de Faitelson-Weiser que comentaré a continuación. Esto se debe a que el corpus que se maneja en los tres casos es diferente. Mighetto y Rosengren trabajan sobre textos de prensa, De la Campa sobre la vigésima edición del DRAE y Faitelson-Weiser sobre un total de dieciséis diccionarios (con caudal hispanoamericano).

\subsection{DiCCIONARIO INVERSO DEL ESPAÑOL. SU USO EN EL AULA, DE H. DE LA CAMPA (1987)}

Este diccionario se compone de 574 páginas y contiene 93.230 vocablos del DRAE, edición 20a (1984). Curiosamente, ha sido confeccionado manualmente con la ayuda de doscientos dieciséis alumnos de bachillerato de Úbeda y Écija, como un trabajo de animación escolar. Es de todos los publicados hasta su momento el de más fácil manejo.

El diccionario de Hermenegildo de la Campa comienza con un prólogo muy interesante que, en primer lugar, nos define un diccionario inverso como:

[...] la ordenación del léxico de una lengua mediante el criterio alfabético, empezando por la última letra de cada vocablo hasta la primera. Con lo cual las palabras se presentan agrupadas por terminaciones iguales. 
Así, pues, nuestro Diccionario Inverso del español empieza por los vocablos $a, a b a, b a b a, a b a b a$, etc. y termina con las palabras rabazuz, cazuz, alcazuz, orozuz.

En segundo lugar, distingue entre los diccionarios de la rima y los diccionarios inversos, dos productos lexicográficos distintos tanto en la forma de distribuir las unidades como en la finalidad que persiguen:

Pues un diccionario de la rima agrupa las palabras sólo a partir de la última vocal acentuada y dentro de cada grupo las palabras son ordenadas por sus comienzos, con lo que las desinencias quedan desordenadas. Por ejemplo, bajo la terminación -ón comienza con abarcón, abazón, abdicación, abducción, abejarrón, etc., con lo que los sufijos tan importantes como -ción, -sión, y -azón, quedan desperdigados.

En tercer lugar, sitúa los antecedentes de los diccionarios inversos en el interés de los estudiosos por la reconstrucción de textos antiguos en lenguas clásicas. De ahí que, como ya he comentado, los primeros diccionarios inversos se hicieran sobre lenguas muertas y hasta 1957 no se publicara el primero sobre una lengua moderna.

En cuanto a las fuentes y criterios de confección, el Diccionario Inverso del español transcribe el léxico de la edición vigésima del DRAE con los siguientes pautas:

1) Se ha respetado la ortografía del DRAE, aun en casos discordantes.

2) En contra de esta edición del DRAE, se ha disuelto la $c h$ y la $l l$ en sus dos grafemas respectivos.

3) Se han registrado los adjetivos en sus dos géneros cuando así lo hace el DRAE.

4) Los sustantivos y adjetivos se introducen sólo en singular, aunque se han consignado en plural cuando el DRAE advierte que el término se usa más de esta forma.

5) Se incluyen las entradas compuestas de más de una palabra.

6) Se excluyen las entradas cuyo final no está completo (prefijos y componentes iniciales de palabras, como, por ejemplo, pro-). 
7) También se han suprimido los sufijos y componentes finales de palabras, pues estas terminaciones están representadas en las terminaciones guías con que se encabezan los vocablos.

8) En caso de doble acentuación, se ha tabulado primero el caso en que el acento está más al final.

9) Cuando un vocablo está precedido de un número, éste indica las veces en que el término está incluido separadamente en el DRAE.

Una novedad que presenta este diccionario inverso con respecto a los anteriores es que divide el repertorio léxico en bloques de terminaciones, con el fin de facilitar la búsqueda de las palabras, el establecimiento de comparaciones y el uso en la enseñanza. Las desinencias se señalan a modo de epígrafes que se acompañan de un número que indica la cantidad de vocablos que incluye. Gracias a este sistema se ha superado la dificultad que suponía recorrer largos listados hasta encontrar el término deseado. Por ejemplo, las palabras terminadas en $-b$ son sólo dieciséis, por tanto, no hay subdivisiones:

$\begin{array}{rrrr}\mathrm{b} & \text { baobab } & \text { jatibal } & \text { chub } \\ \mathrm{ab} & \text { salab } & \text { coulomb } & \text { club } \\ \mathrm{abab} & \text { mihrab } & \text { Job } & \text { querub } \\ \text { nabab } & \text { Deneb } & \text { rob } & \text { sub }\end{array}$

Sin embargo, las acabadas en - $a$ son 30.830 , de las cuales 235 terminan en - $b a$ (las cuales se distribuyen bajo las terminaciones - $a b a$, -sila$b a$, etc.).

$\mathrm{Al}$ final del diccionario se incluye un esquemático Anexo Estadístico que comprende:

1) Relación de grafemas finales de palabras presentados alfabéticamente.

2) Relación de grafemas finales de palabras presentados por orden decreciente de su frecuencia.

3) Grafemas vocálicos y consonánticos.

Tras el prólogo, el Diccionario Inverso del español presenta una in- 
troducción práctico-didáctica que da cuenta de las utilidades del diccionario, las cuales resumo a continuación":

1) Utilidad lúdica para los aficionados a crucigramas, damero y demás juegos de palabras, ya que sirve para enriquecer el vocabulario y localizar palabras con una determinada terminación y extensión.

2) Utilidad publicitaria, pues el diccionario puede ayudar a encontrar palabras apropiadas para un eslogan sugerente o un título de reclamo.

3) Utilidad versificadora, puesto que, además de la rima consonante, el diccionario proporciona la rima rica, es decir, aquella en la que coinciden también los fonemas anteriores a la vocal tónica. Además, aporta la posibilidad de hallar toda la gama de fonemas verbales ${ }^{10}$.

4) Utilidad ortográfica, ya que el diccionario inverso facilita el aprendizaje de la ortografía y la investigación de nuevas técnicas para dominarla. Gracias a la estadística observamos la rentabilidad de determinadas reglas.

5) Utilidad etimológica y semántica, ya que, al aprender los vocablos por bloques etimológicos y semánticos, se potencia el dominio del léxico.

6) Utilidad investigadora, sobre todo, en los campos de la morfolo-

9 Sobre las aplicaciones didácticas de estos diccionarios véanse los trabajos de Hermenegildo de la Campa et al. (1990a, 1990b, 1992, 1994-1995). Las ideas de estos artículos sobre las utilidades didácticas de los diccionarios inversos se recogen también en las introducciones de los tres diccionarios inversos que ha dirigido el profesor de la Campa $(1987,1991,1996)$.

10 Así explica de la Campa (1987: 16) las ventajas del diccionario inverso con respecto al de la rima, tomando como ejemplo la rima consonante -ira: "el diccionario de la rima me proporcionará todos los nombres con esa terminación (lira, pira, etc.), pero no las formas verbales con esa desinencia, como son los presentes y los imperativos en ira (como admira, suspira, expira, etc.), fácilmente encontrables bajo -irar en nuestro diccionario y que son 29, pero que no se encontrarán agrupados en el de la rima porque la -i- de -irar es átona, por lo que -irar va allí mezclado con la terminación tónica - $a r$, o sea, la del infinitivo más común, en un océano de vocablos, desordenados además según sus terminaciones. Tengamos presente que en nuestro diccionario las palabras terminadas en -ar son 9.360". 
gía derivativa, fonología, lingüística computacional y estudio comparativo de las lenguas.

7) Utilidad docente, para medir el vocabulario pasivo de los alumnos o para obtener de una manera rápida los vocablos derivados de uno primitivo.

\subsection{DICTIONNAIRE INVERSE ET ANALYSE STATISTIQUE DE LA LANGUE ESPAGNOLE DE S. FAITELSON-Weiser (1987)}

El DIASLE, como se conoce de forma abreviada el Diccionario inverso y análisis estadístico de la lengua española, se comenzó a realizar en 1958, dos años más tarde de la publicación del Reverse Dictionary of the Spanish Language e incluye caudales léxicos hispanoamericanos. Es una obra muy extensa que contiene numerosos análisis estadísticos ${ }^{11}$. El diccionario se adjunta al libro en seis microfichas (que equivalen a 1.231 páginas a dos columnas). Se trata, por tanto, de un trabajo dirigido a investigadores y especialistas, pues necesita de un aparato lector de microfichas.

El diccionario va precedido de una introducción trilingüe (francés, español, inglés) que consta de una Presentación General, en la que se explica que, a diferencia del diccionario de Stahl y Scavnicky, que no toma en cuenta el acento de las palabras, en éste se separan las vocales tónicas de las átonas, ya que en español el acento posee un valor fonológico.

En cuanto al caudal léxico que se emplea, este diccionario inverso, en un intento de ser lo más representativo posible, recoge, tras haber examinado quinientos diccionarios españoles, el léxico de un total de dieciséis $^{12}$, incluida la edición del DRAE de 1970 acompañada de las

11 El índice y el prólogo del DIASLE abarcan veinticuatro páginas y la introducción doscientas sesenta y una.

12 Las fuentes son: DRAE (1970); Larousse (1978): Dictionnaire moderne fraçais-espagnol / espagnol-français; F. J. Santamaría (1942): Diccionario general de americanismos; E. Rodríguez Herrera (1958-1959): Léxico mayor de Cuba, La Habana, 2 vols.; A. Santamaría, A. Cuartas and J. Mangada (1975): Diccionario de incorreccio- 
correcciones y adiciones que figuran en el Boletín de Academia (99.229 entradas). Para obtener esta selección de obras lexicográficas se tuvieron en cuenta las más modernas de cada país y región, los diccionarios de americanismos más recientes y los últimos diccionarios generales del español. Estos fueron sometidos a un test estadístico que mostró cuáles, proporcionalmente a su extensión, contenían el mayor número de formas ausentes del DRAE.

Con todo, las palabras obtenidas sumaban un total de 289.408 , de las que se han tomado 180.977 , ya que se han dejado fuera las de origen extranjero que, aunque estaban recogidas en las fuentes lexicográficas, planteaban problemas específicos de ortografía o pronunciación. El corpus final incluye voces de distintas regiones hispanoamericanas, variantes sociales, fonéticas y estilísticas.

Otra diferencia clave con respecto al diccionario de Stahl y Scavnicky, es que se emplean indicaciones gramaticales, de hecho cada palabra va acompañada de una abreviatura, que indica su categorización, y de una serie de números, que representan las fuentes lexicográficas en las que ha sido encontrada. Estos números a veces van marcados con un asterisco que indica que la palabra se encuentra en el suplemento de la fuente en cuestión. Los números pueden presentarse también en letra negrilla, itálica o itálica negrilla, con lo que hacen referencia a tres de los apéndices que se incluyen en el diccionario para dar cuenta,

nes, particularidades y curiosidades del lenguaje, Madrid; J. V. Solá (1950): Diccionario de regionalismos de Salta, Buenos Aires; R. A. Galván y R. V. Teschner (1975): El diccionario del español de Tejas. The Dictionary of the Spanish of Texas (Spanish-English), Silver Spring; A. Cordero Palacios (1957): Léxico de vulgarismos azuayos, Cuenca; E. D. Tovar (1966), Vocabulario del oriente peruano, Lima; Academia colombiana de Lexicografía (1975): Breve diccionario de colombialismos, Bogotá; N. Fernández Naranjo y D. Gómez de Fernández (1967): Diccionario de bolivianismos (con un suplemento de ocho apéndices), La Paz; J. Cáceres Freyre (1961): Diccionario de regionalismos de la Provincia de la Rioja, Buenos Aires; C. J. Cela, Diccionario secreto (1968): Madrid-Barcelona; L. Aguilera Patiño (1951): "Diccionario de panameñismos", BAAL, Buenos Aires; E. A. Doty (1958): A Glossary of the Filipinismos in the Spanish Language Found in the Publications of the Period 18901920, Michigan; S. de Lugo (1946): Colección de voces y frases provinciales de Canarias, La Laguna. 
respectivamente, de las palabras que presentan modificaciones gráficas, diferencia de categorización o que aparecen bajo una rúbrica diferente con respeto a su fuente.

Las palabras han sido clasificadas en doce categorías (sustantivos, adjetivos, verbos, adverbios, determinantes, pronombres, preposiciones, conjunciones, interjecciones, contradicciones y palabras ligadas ${ }^{13}$ ).

Aunque los términos se introducen sin flexionar, esto es, los verbos en infinitivo, los sustantivos y adjetivos en singular, etc., se incluyen las formas diferentes en cuanto a grafía y pronunciación de una misma palabra (así de los determinantes y pronombres se registran los femeninos y plurales, algunos casos de diminutivos, aumentativos y superlativos, ciertas formas de plural que presentan un significado distinto con respecto al singular, etc.).

Una característica muy importante de este diccionario es que nos proporciona toda una serie de cuadros con las frecuencias absolutas y relativas, además, nos presenta contrastados los finales del francés, del español y del italiano. Los resultados en cuanto a los finales del español varían con respecto a los que nos proporciona Hermenegildo de la Campa; esto se debe a que el diccionario de Faitelson-Weiser maneja el doble de vocabulario y a que no cuenta grafemas sino fonemas, con la intención de que queden juntos los homófonos y no los homógrafos. Este hecho, aunque enriquecedor para estudiar la lengua española desde una nueva perspectiva, dificulta mucho la búsqueda de palabras, ya que éstas se encuentran ordenadas según la pronunciación (de forma que aparecen juntas $i$ e $y$; $b$ y $v ; c$ oclusiva, $k$ y $q$, etc.); los fonemas diferentes que no representan más que uno en determinadas variedades se han ordenado separados pero uno detrás de otro (así ocurre con $y$ y $l l ; y$ con $z, c$-ante $e, i-\mathrm{y} s$ ); además, en el orden alfabético seguido se introducen primero las tónicas y luego las átonas. Así, en primer lugar, aparecen los signos que no se pronuncian, seguidos de la /a/ (tónica y átona), los fonemas anteriores (primeros los vocálicos y luego los

13 El diccionario considera palabras ligadas a aquéllas que sólo se emplean como componentes de una frase, de una locución o de una expresión dada. 
consonánticos), los fonemas posteriores (comenzando también por las vocales), siguiendo con las consonantes velares.

En general, las palabras conservan su ortografía habitual en español, aunque en algún caso se ha variado la acentuación, como en determinados neologismos de origen extranjero.

\subsection{DicCionaRio INVERSO DE LA LENGUA ESPAÑOLA DE I. BOSQUE Y M. PÉREZ FERNÁNDEZ (1987)}

Publicado por una editorial de prestigio, este diccionario inverso es el más conocido. En el prólogo, se diferencian los diccionarios inversos de los de la rima en tanto que estos -en su origen-no estaban confeccionados por procedimientos mecánicos. Además su realización tampoco es idéntica. El prólogo explica la importancia del diccionario inverso para las diferentes disciplinas lingüísticas, como puede ser la morfología derivativa. Así, por ejemplo, sirve para saber cuántas voces terminadas en -mente posee el español. También es una herramienta de trabajo para los especialistas en fonología, en métrica, en lingüística computacional y, en particular, en el estudio de la estadística léxica. Es práctico, además, para la lingüística comparativa y los estudios de lexicología contrastiva, ya que existen diccionarios inversos en diferentes lenguas romances. No deja de ser importante para los publicistas, los interesados en el lenguaje poético y en la versificación, en pasatiempos basados en el léxico, etc. ${ }^{14}$

El diccionario incluye 102.012 voces que provienen de la vigésima edición del DRAE de 1984 y del Diccionario crítico etimológico castellano e hispánico de Corominas y Pascual (1980). Recoge también voces antiguas y dialectales -puesto que el DRAE lo hace-, así como voces del castellano medieval, las cuales mantienen las grafías originales en los casos en que Corominas las mantiene ${ }^{15}$.

14 Como se comenta en el prólogo, el diccionario inverso del ruso que se preparó en Alemania durante la Segunda Guerra Mundial sirvió de gran ayuda para descifrar los mensajes en clave. Al menos así lo comenta Jitka Stindlova (1960: 79).

15 Verd (1993: 101) ha señalado como un defecto de este diccionario esta mezcla de 
Los términos se han distribuido en tres columnas de cincuenta palabras por páginas. No se recogen los diminutivos y aumentativos. Se admiten los superlativos irregulares y los que poseen valores significativos propios. El criterio que sigue el DRAE en este aspecto es el de evitar dar entrada a las voces formadas con afijos productivos cuando no existen irregularidades y se obtienen resultados predecibles. Sin embargo, esto no se aplica a las voces terminadas en -ble o -mente, que se introducen sistemáticamente, aunque cumplan las condiciones señaladas. Tanto en este diccionario como en el DRAE se observan numerosos términos en -ificar y escasísimos en -ificador.

Como en el DRAE, se han tomado nombres propios que forman parte de unidades léxicas complejas o de acuñaciones idiomáticas. En tales casos, el nombre propio suele aparecer en una frase prepositiva que identifica un tipo o una variedad de la entidad designada por el núcleo del sintagma al que modifica. Se trata de unidades léxicas complejas en la que aparecen nombres propios desprovistos de su valor designativo como topónimos o antropónimos.

En este diccionario se han mantenido los criterios usados por el DRAE -a pesar de que se ha percibido cierta inestabilidad en ellos- para escribir con mayúscula o minúscula determinadas voces y para conceder, o no, lugar a otras considerándolas como nombres propios.

Se mantienen las grafías dobles (con y sin guión) de las palabras compuestas cuando el DRAE recoge ambas formas (judeo-español y judeoespañol, tiquis miquis y tiquismiquis), así como algunas entradas del diccionario académico que constan de unidades léxicas repetidas ( $h u h u h u$, je je je, etc.), debido a que se considera que la iteración de sus componentes es un fenómeno morfológico y no sintáctico.

Se han suprimido -aunque el DRAE las mantiene- las expresiones léxicas compuestas, de valor designativo formadas por procedimientos sintácticos (cochinillo de San Antón, bran de Inglaterra,...). Asimismo, se han omitido las locuciones adverbiales, conjuntivas o preposiciona-

niveles heterogéneos de lengua que, en su opinión, deberían ser tratados separadamente. 
les (pese a, herre que herre,...), pues hubieran sido perjudiciales para las estadísticas léxicas.

Los latinismos introducidos por el DRAE se han consignado en el diccionario, así como los prefijos, sufijos y bases léxicas ligadas (archi, alo-, fisio-, -forme-, -avo, -algia,...).

Aunque los adjetivos de dos terminaciones se recogen en el DRAE en la misma entrada, en este diccionario inverso se consideran entradas independientes.

Contra el criterio del DRAE, las grafías dobles $(c h, l l, r r)$ no se han considerado como letras únicas, sino independientes, solución que parece haber augurado los criterios posteriores de la Academia.

Dado que este diccionario inverso incluye muchos términos antiguos e incluso respeta su grafía, la letra $c ̧$, considerada independiente, va ordenada tras la $c$, facilitando así su localización.

Para terminar, destaca -como única especificación- el uso del asterisco que acompaña a algunas voces ${ }^{16}$. Como ejemplo, veamos las incluidas en la letra -B, que si se cotejan con las extraídas del Diccionario Inverso del español de Hermenegildo de la Campa, observamos que, de dieciséis voces, se ha aumentado a veintidós:

$\begin{array}{rrr}\text { b } & \text { B } & \text { job } \\ \mathrm{ab} & \text { axarab* } & \text { rob } \\ \mathrm{abab} & \text { mihrab } & \text { alchub } \\ \text { nabab } & \text { almutaceb* } & \text { club } \\ \text { baobab } & \text { Deneb } & \text { querub } \\ \text { cab* }_{\text {quiçab* }}^{*} & \text { algib* } & \text { sub- } \\ \text { salab } & \text { jatib } & \end{array}$

16 Las 8.040 voces que van marcadas con asterisco están todas extraídas del diccionario de Corominas y Pascual y no figuran en la vigésima edición del DRAE. 


\subsection{PROYECTO ONE71. BANCO DE DATOS DE ONCE NOVELAS ESPAÑOLAS 1951-1971. LISTA REVERSA DE PALABRAS GRÁFICAS CON FRECUENCIA TEXTUAL DE D. MighetTo y P. Rosengren (1988)}

Consta de 212 páginas. Se trata de un diccionario inverso especializado, pues si el Diccionario reverso publicado por estos autores en 1985 versaba sobre el léxico de la prensa, este segundo proyecto se basa en un millón de palabras (exactamente 1.005.012) obtenidas de once novelas españolas ${ }^{17}$.

El trabajo consta de la concordancia (81 microfichas), el texto seguido separado por novelas (11 microfichas), una lista de frecuencias de palabras gráficas (editada en 5 microfichas y en listado de computadora) y una lista inversa de palabras gráficas que van acompañadas de cifras que señalan su frecuencia textual (212 páginas en listado de computadora).

Resulta un estudio muy interesante en cuanto que la frecuencia textual de las palabras nos ayuda a caracterizar el lenguaje literario de una época concreta. Además ofrece una fuente atractiva para investigadores de lingüística española y para la enseñanza del español como segunda lengua.

\subsection{DiCCIONARIO INVERSO ESPAÑOL-COREANO DE WOO-JOONG (1991)}

Se trata de otro tipo de diccionario inverso especializado, pues va dirigido a estudiantes de español como segunda lengua, por lo que cada término va acompañado de una breve traducción al coreano.

17 Según explican los autores en la introducción: "Ocho de estas novelas pertenecen a la época del realismo "social" de la novela española (1951-1962) y son: La Colmena (Cela 1951), La noria (Romero 1952), Juegos de manos (Goytisolo 1954), El Jarama (Sánchez Ferlosio 1956), Ayer, 27 de octubre (Olmo 1958), Central eléctrica (López Pacheco 1958) y Tormenta de verano (García Hortelano 1962). Las tres restantes corresponden al tiempo que va entre el agotamiento del social-realismo y la llamada renovación experimentalista de la novela española (1972-1975); las mismas son: Las últimas banderas (De Lera 1967), Últimas tardes con Teresa (Marsé 1967), y Torremolinos Gran Hotel (Palomino 1971)". 
Consta de 924 páginas y ha sido realizado por un grupo de alumnas universitarias, que durante cuatro años han papeleteado el vocabulario a mano. Presenta 60.000 palabras seleccionadas de la vigésima edición del DRAE (1984) y otras fuentes. Va precedido de un prólogo bilingüe y termina con unos cuadros estadísticos. En el breve prólogo el autor, además de los agradecimientos y de hacerse cargo de los posibles errores, da cuenta de la utilidad de la obra:

Siempre que observe cualquier cosa al revés o contrariamente, percibimos tales cosas como algo nuevo y entonces muchas de esas cosas que antes habíamos ignorado se nos aparecen claras y lógicas. La presente obra es el resultado pues de esta 'observación contraria'. Con este diccionario inverso podemos observar el 'otro mundo' del lenguaje y descubrir la 'otra cara' léxica del español que está escondida en los diccionarios ordinarios.

Tengo convicción de que esta obra será de gran utilidad tanto para profesores y versificadores como para alumnos y algunos profesionales interesados en el lenguaje. Sobre todo será sumamente valioso como texto de vocabulario español porque se ha puesto especial cuidado en potenciar el dominio del léxico.

Este diccionario se caracteriza porque es una especie de colección de diccionarios inversos, pues presenta separados los sustantivos, adjetivos, verbos, pronombres, preposiciones, adverbios, conjunciones, interjecciones y onomatopeyas. Aquéllas que pertenecen a dos categorías se incluyen en las dos secciones correspondientes. Se señalan los sustantivos con distintas acepciones en el plural, los plurales peculiares, las formas de los verbos irregulares, se añaden indicaciones sobre etimología, género, etc. Se incluye, además, un diccionario inverso de todos los esdrújulos, agrupados por sus rimas. Al final aparece una serie de palabras agrupadas por su origen.

En el orden alfabético seguido se consideran letras simples los dígrafos $c h$, la $l l$ y la $r r$.

En los listados de palabras se señalan con negrita los finales que se repiten para indicarnos las palabras emparentadas. Si tienen la misma terminación pero distinta etimología, se distinguen la de cada familia mediante asteriscos. 
3.9. DicCIONARIO ESCOLAR INVERSO DE LA LENGUA ESPAÑOLA. IMPLICACIONES DIDÁCTICAS, DE H. DE LA CAMPA, E. DE MANUEL, A. ROMERO Y F. ROMERO (1991)

Este diccionario sigue la línea iniciada por Hermenegildo de la Campa en el año 1987. El trabajo consta de 314 páginas divididas en un sumario en interlingua, un prefacio y una extensa introducción en la que se resumen los antecedentes de estos diccionarios, las fuentes, los criterios de elaboración, el método de trabajo seguido y las implicaciones didácticas para el estudio del léxico en la enseñanza básica (utilidad creativa y lúdica, versificadora, semántica, ortográfica e investigadora).

Tras las referencias bibliográficas, sigue el diccionario escolar inverso, que presenta los epígrafes -de terminaciones y de comienzos de palabras- acompañados con el número de vocablos que recogen, lo que facilita la búsqueda de los términos. Después del diccionario inverso se introduce un vocabulario básico de la educación obligatoria y, finalmente, se ofrecen una serie de cuadros estadísticos ${ }^{18}$.

Lo característico de este diccionario es que se trata de un diccionario inverso específico en cuanto que está orientado a la escuela. Contiene 13.777 vocablos tomados de un vocabulario básico para la escolaridad obligatoria (éste se incluye tras el diccionario inverso). El vocabulario se ha obtenido a partir de ocho diccionarios escolares ${ }^{19}$ y de la vigésima edición del diccionario académico (1984). Además se han consultado once estudios sobre vocabulario ${ }^{20}$.

18 El diccionario escolar inverso ocupa 142 páginas y el vocabulario básico 120.

19 Mi primer diccionario, ed. Everest (1980); Brocal. Vocabulario básico ilustrado, La Galera (1981); Diccionario escolar etimológico, Magisterio Español (1985); Vox. Diccionario inicial de la lengua española, Bibliograf (1986); Diccionario escolar lengua española I, II, III, Everest (1987); Diccionario básico de la lengua, ed. Anaya (1989); Espasa Escolar, Espasa (1989); Larousse Junior. Diccionario de la E.G.B., Larousse (1989).

20 Rodríguez Bou y colaboradores (1952): Recuento de vocabulario español, Universidad de Puerto Rico; García Hoz (1953): Vocabulario usual, común y fundamental, C.S.I.C., Madrid; Juilland y Chang-Rodríguez (1964): Frequency Dictionary of Spanish Words, Mouton and Co., London; Armayor (1975): Determinación del vocabula- 
Es, por tanto, un diccionario bastante más reducido que el publicado por de la Campa en 1987 (con 93.230 vocablos). Baste, como ejemplo, comentar que de las 30.830 palabras acabadas en - $a$ que se introdujeron en aquél ahora se recogen 4.545. Dentro de este grupo en la terminación -dica se incluían 55 voces que ahora han quedado fijadas en 7:

$$
\begin{array}{r}
\text {-dica } \\
\text { sádica } \\
\text { médica } \\
\text { ortopédica } \\
\text { verídica } \\
\text { jurídica } \\
\text { periódica } \\
\text { nórdica }
\end{array}
$$

\subsection{DicCionario inVERSo de Fontanillo Merino y Riesco (1992)}

Formando parte del volumen octavo de un Diccionario enciclopédi$c o$, se publica el Diccionario inverso de Enrique Fontanillo y $\mathrm{M}^{\mathrm{a}}$ Isabel Riesco, basado en la decimonovena edición del DRAE. Llama la atención que en el prólogo se confunden los diccionarios de la rima con los inversos, se considera a aquellos los predecesores de estos y se fecha el

rio fundamental (de uso y reconocimiento) del niño cordobés de seis años, en su incorporación a la E.G.B., I.C.E., Universidad de Córdoba; Armayor (1977): Determinación del vocabulario fundamental (de uso y reconocimiento) del niño cordobés de catorce años, en último curso de la E.G.B., y su incorporación al B.U.P. I.C.E., Universidad de Córdoba; Armayor (1979): Determinación del vocabulario técnico escolar en el alumno de $8^{\circ}$ de E.G.B. y su incorporación al B.U.P., I.C.E., Universidad de Córdoba; Díaz Castañón, Domingo García, Blázquez Fabián et al. (1977): Vocabulario básico del español y sus aplicaciones a la enseñanza I, I.C.E., Universidad de Oviedo; Díaz Castañón y Domingo García (1978): Vocabulario básico del español y sus aplicaciones a la enseñanza II, I.C.E., Universidad de Oviedo; Justicia Justicia (1985): El vocabulario usual del niño en el Ciclo Inicial y el Ciclo Medio de la E.G.B., I.C.E., Universidad de Granada; Casanova Rodríguez y Rivera González (coord.) (1989): Vocabulario básico en la E.G.B., 3 vols., Ministerio de Educación y Ciencia, Espasa Calpe, Madrid; Guerra Salas (1989): Comprensión y producción del léxico básico en Enseñanzas Medias, Tesis doctoral, Universidad Complutense de Madrid. 
nacimiento de los inversos en el siglo pasado, cuando, como ha demostrado Verd, existen desde el $\mathrm{XVI}^{21}$.

Incluye 82.764 vocablos, que se distribuyen en cinco columnas siguiendo un orden alfabético estricto. Se trata de un listado continuado, sin ningún tipo de información adicional. La búsqueda de las palabras resulta dificultosa, pues la única guía es una terminación en negrita que, en el extremo superior izquierdo, indica la terminación de la primera palabra de la página y, en el extremo superior derecho, indica la terminación de la última.

Se trata, por tanto, de un diccionario un tanto anacrónico, ya que, a pesar de su fecha de publicación (1992), no sólo sigue una edición del DRAE lejana (1970), sino que mantiene las características de los primeros diccionarios inversos, pues, como el Diccionario de terminaciones de Martínez y Martínez de Carnero (1978), se trata de un listado continuado, sin ningún tipo de información adicional ni facilidad en las búsquedas.

\subsection{DicCIONARIO INVERSO DE LA POESÍA DE QUEVEDO, DE S.}

Fernández Mosquera y A. Azaústre Galiana (1993)

Se trata de un diccionario inverso muy específico, ya que está realizado sobre un corpus muy limitado, la poesía de Francisco de Quevedo. El diccionario está incluido en los Índices de la poesía de Quevedo, obra realizada por Santiago Fernández Mosquera y Antonio Azaústre Galiana. El diccionario ocupa 166 páginas de las 1.053 que componen los Índices. Para su realización, los autores se han ayudado del programa dBASE IV de ASHTON TATE. Han utilizado los vocablos incluidos en los textos de la poesía de Quevedo considerados BASE por Blecua $(1969,70,71,81)$, cuya edición se sigue en la realización de los índices. Como los autores indican en la introducción, el diccionario inverso "puede ser de gran utilidad para consultas de tipo lingüístico y para el estudio de las rimas, al ordenar alfabéticamente las palabras por su terminación" (1993:15).

21 Cfr. nota 3. 
La única característica de este diccionario es que cada término va acompañado de un número que indica el número de veces que se repite en la poesía de Quevedo.

\subsection{DICCIONARIO INVERSO DEL VOCABULARIO ESCOLAR BÁSICO: \\ IMPLICACIONES DIDÁCTICAS, DE H. DE LA CAMPA, A. ROMERO LÓPEZ, F. ROMERo LÓPEZ Y J. LÍNDEZ CAZORLA (1996)}

Como los propios autores comentan en el prefacio, este diccionario -que consta de 447 páginas- supone la maduración del Diccionario Escolar Inverso de la Lengua Española. Implicaciones didácticas, publicado cuatro años antes.

Tras el prefacio de los autores, se reproduce prácticamente la rica introducción del diccionario publicado en 1991 (antecedentes, fuentes, criterios de elaboración, métodos de trabajo seguidos e implicaciones didácticas $)^{22}$.

Las fuentes que se han tenido en cuenta para la elaboración de este diccionario han sido las mismas que las usadas en el diccionario de 1991: ocho diccionarios escolares, infantiles y juveniles ${ }^{23}$ y once trabajos sobre vocabulario ${ }^{24}$. No obstante, la edición del DRAE que se sigue es la vigésimo primera (1992).

La innovación que presenta este diccionario con respecto al Diccionario Escolar Inverso de la Lengua Española. Implicaciones didácticas, es que el vocabulario básico -que ocupa 166 paginas- ha sido revisado, corregido y fijado en 11.781 palabras, que son las que los autores consideran que debe conocer el alumno al llegar a los catorce años. Este vocabulario precede al diccionario inverso, que recopila un total de 13.999 términos distribuidos en 216 páginas.

Además, el diccionario consigna la categoría gramatical de los tér-

22 La única variación parece estar en el método de trabajo, pues se ha utilizado para la ordenación alfabética de las palabras un programa más moderno.

23 Cfr. nota 19.

24 Cfr. nota 20. 
minos. Pero lo más novedoso es que señala si el vocablo es internacional o no. Veamos como ejemplo la terminación -hada, que se incluye dentro de los 332 vocablos en - $a d a^{25}$.

$$
\begin{array}{r}
\text {-hada } \\
\text { hada= s.f. } \\
\text { fachada= s.f. } \\
\text { aprovechada\#adj. } \\
\text { desdichada\#adj. } \\
\text { manchada\#adj. } \\
\text { almohada\#s.f. }
\end{array}
$$

Hasta aquí una breve descripción de doce diccionarios inversos impresos en lengua castellana. Seguramente deben existir más, bien integrados como elementos auxiliares en otros trabajos impresos (similar al diccionario inverso incluido en los Índices de la poesía de Quevedo que he comentado) o en disquetes, como ocurre en el CLAVE. Diccionario de uso del español actual (1997), que incluye en el CD Rom un diccionario inverso.

\section{BIBLIOGRAFÍA}

\section{A) Diccionarios}

BosQue, I. y M. PÉREZ FERnÁNDEZ (1987): Diccionario inverso de la lengua española, Madrid, Gredos.

CAMPA, H. de la (1987): Diccionario Inverso del español, Su uso en el aula, Madrid, Narcea.

- - E. de MANuel, A. Romero, F. Romero (1991): Diccionario escolar inverso de la lengua española. Implicaciones didácticas, Granada, Universidad, Instituto de Ciencias de la Educación (ICE).

CAMPA, H. de la (dir.), A. ROMERo LóPEZ (coord.), F. ROMERo LóPEZ, J. LínDeZ CAZORLA, J. (1996): Diccionario inverso del vocabulario es-

25 Se entiende que un vocablo es internacional si existe en tres o más de las lenguas siguientes: francés, inglés, italiano, alemán, español-portugués y ruso. En el diccionario el término internacional va acompañado del signo =, el que no lo es del signo \#. La abreviatura $s$ se refiere a sustantivo y $f$ a femenino. 
colar básico: implicaciones didácticas, Granada, Fundación Educación y Futuro.

CLAVE. Diccionario de uso del español actual (1997), Madrid, SM, ed. en CD Rom.

Corominas, J. y J. A. Pascual (1980): Diccionario crítico etimológico castellano e hispánico, Madrid, Gredos.

FAITELSON-WEISER, S. (1987): Dictionnaire inverse et analyse statistique de la langue espagnole, Québec, Université Laval.

FontANILLO, E. y M ${ }^{\mathrm{a}}$ I. Riesco (1992): Diccionario inverso, $2^{\mathrm{a}}$ parte del vol. 8 del Diccionario enciclopédico, Barcelona, Plaza y Janés.

LeChado García, J. M. (2000): Diccionario de eufemismos y de expresiones eufemísticas del español actual, Madrid, Verbum.

MARTíNEZ Y MARTÍNEZ DE CARNERO, J. J. (1978): Diccionario de terminaciones. Extraído del de la Real Academia Española, Logroño, edición del autor.

Mighetto, D. y P. RosengRen (1985): Diccionario reverso, Göteborg, Universitet, Institutionen för Romanska Språk.

(1988): Proyecto ONE71, Banco de Datos de Once Novelas Españolas 1951-1971. Lista reversa de palabras gráficas con frecuencia textual, Gotemburgo, Dep. de Lenguas Romances, Universidad de Gotemburgo.

StAhL, F. A. y G. E. A. SCAVNICKy (1973): A Reverse Dictionary of the Spanish Language, Urbana, Chicago, University of Illinois Press.

V.V.A.A. (1997): Diccionario inverso ilustrado. De la idea aproximada a la palabra precisa, Madrid, Reader's Digest Selecciones.

Woo-Joong, K. (1991): Diccionario inverso español-coreano, Hyosung, Universidad femenina de Hyosung.

B) EsTUDIOS

BleCuA, J. M. (ed.) (1969, 1970, 1971, 1981): Quevedo, Obra poética, 4 vols., Madrid, Castalia.

CAMPA, H. de la et al. (1990a): "Los diccionarios inversos y sus implicaciones didácticas para la enseñanza de la lengua en educación básica: utilidades semántica y ortográfica", en Actas del I Congreso de la Sociedad Española de Didáctica de la Lengua y la Literatura, Sevilla, Departamento de Didáctica de la Lengua y la Literatura y Filologías Integradas de la Escuela Universitaria de Magisterio, pp. 109-113. 
(1990b): "Algunas implicaciones didácticas de los diccionarios inversos para la enseñanza de la lengua en educación básica: utilidades creativa y lúdica, versificadora e investigadora”, Revista de Educación de la Universidad de Granada, 4, pp. 25-39.

(1992): "La enseñanza del vocabulario en el aula mediante la utilización del diccionario escolar por familias etimológicas", Revista de Educación de la Universidad de Granada, 6, pp. 67-77.

(1994-1995): "Los diccionarios escolares inversos: su utilidad para la enseñanza y el aprendizaje del español como lengua materna y como lengua segunda", Revista de Educación de la Universidad de Granada, 8, pp. 281-285.

Casas Homs, J. M. (ed.) (1962): La Gaya Ciencia, 2 vols., transcripción de O. J. Tuulio, Madrid, CSIC.

Fernández Mosquera, S., y A. AZAústre Galiana (1993): Índices de la poesía de Quevedo, Barcelona-Santiago de Compostela, PPU-Universidad de Santiago de Compostela.

GUERRERo SALAZAR, S. (2001a): "Los diccionarios de la rima y los diccionarios inversos españoles: afinidades y diferencias", en A. M. Medina Guerra (coord.), Estudios de lexicografía del español ( $V$ Centenario del Vocabularium ecslesiasticum de Rodrigo Fernández de Santaella), Málaga, Universidad de Málaga, pp. 317-340.

(2001b): "Los diccionarios de la rima españoles hasta el siglo XIX: análisis y evolución histórica", Letras de Deusto, 31, n 92, pp. 4570.

(2002): "Los diccionarios de la rima españoles en el siglo XX", Letras de Deusto, 32, n 94, pp. 29-41.

HAENSCH, G. (1997): Los diccionarios del español en el umbral del siglo $X X I$, Salamanca, Universidad de Salamanca.

MigheTto, D. y P. Rosengren (1982): Banco de datos de Prensa Española: Concordancia lingüística y texto fuente, Göteborg, Universitet, Institutionen för Romanska Språk.

(1983): Palabras Gráficas Españolas: Lista y frecuencia en Prensa Española, 1977, Göteborg, Universitet, Institutionen för Romanska Språk.

StindLovA, J. (1960): "Sur le classement inverse des mots et sur ce qu'on appelle dictionnaire inverse", Cahiers de lexicologie, 2, pp. 79-86.

VERD, G. Ma . (1993): "Sobre los diccionarios inversos y los españoles en particular", Letras de Deusto, 23, n 58, pp. 85-115. 